\title{
Rileggendo Sainte-Beuve: è il "Port-Royal" ad aver 'fatto' la grandezza dei Solitari?
}

\section{Benedetta Papasogli}

\section{Q OpenEdition}

1 Journals

\section{Edizione digitale}

URL: https://journals.openedition.org/studifrancesi/4542

DOI: $10.4000 /$ studifrancesi. 4542

ISSN: 2421-5856

\section{Editore}

Rosenberg \& Sellier

\section{Edizione cartacea}

Data di pubblicazione: 1 avril 2012

Paginazione: 79-83

ISSN: 0039-2944

\section{Notizia bibliografica digitale}

Benedetta Papasogli, «Rileggendo Sainte-Beuve: è il "Port-Royal" ad aver 'fatto' la grandezza dei Solitari?», Studi Francesi [Online], 166 (| | LVI) | 2012, online dal 30 novembre 2015, consultato il 19 novembre 2021. URL: http://journals.openedition.org/studifrancesi/4542 ; DOI: https://doi.org/ 10.4000/studifrancesi.4542

\section{(c) (i) (9)}

Studi Francesi è distribuita con Licenza Creative Commons Attribuzione - Non commerciale - Non opere derivate 4.0 Internazionale. 


\section{Rileggendo Sainte-Beuve: è il "Port-Royal" ad aver 'fatto' la grandezza dei Solitari?}

Il Port-Royal di Sainte-Beuve è stato accolto in Francia nella collana della «Pléiade», in una edizione del 1955 a cura di Maxime Leroy, ed è presente nei «BouquinsLaffont» a cura di Philippe Sellier (2004); risulta dunque essere l'unica opera di Sainte-Beuve che abbia avuto l'onore di un'assunzione nella collana dei grandi classici, e al tempo stesso l'unica che goda oggi di una vasta diffusione nelle librerie attraverso una di quelle collane dotte eppure genialmente leggere che garantiscono l'accessibilità di capolavori fuori formato. In Italia una traduzione del Port-Royal a cura di Serena d'Arbela era apparsa quasi cinquant'anni or sono presso Sansoni (1964). Era la prima, a riprova di quanto l'opera sia stata in casa nostra più citata e rispettata che realmente letta, come suggerisce Mario Richter che ha oggi un merito straordinario: a lui si deve la nuova edizione italiana del Port-Royal nei «Millenni» Einaudi (2011), cioè un ritorno del capolavoro attraverso quella specie d'arco trionfale che è una pubblicazione da tutti i punti di vista preziosa, accuratissima e bella.

Preziosa in primo luogo nell'immagine, e nel suo interno corredo d'immagini. Sappiamo quanto conti un buon apparato iconografico per un approccio alla realtà di Port-Royal. Da una parte le tele austere di Philippe de Champaigne, che da quando ebbe una figlia di là dalla clausura si dedicò a ritrarre i grandi protagonisti della storia del monastero, e del giansenismo: la Mère Angélique, Antoine Arnauld, SaintCyran, Singlin, Barcos... poiché il Port-Royal di Sainte-Beuve è anche una collezione di «portraits littéraires» e una galleria di «hommes / femmes illustres», il dialogo fra il racconto e l'immagine affascina il lettore come un aprirsi della pagina verso il profondo. Dall'altra parte, un materiale meno solenne: le gouaches secentesche che fermano il fuggitivo, sia la sacra conversazione delle moniali sia la loro processione in grandi abiti verso il coro, o l'aspetto dei tetti e degli orti di Port-Royal des Champs. La scomparsa del monastero istituisce di questo patrimonio figurativo, specie se dedicato alla vita ordinaria, la virtù mnemotecnica e la natura di ombra.

Questa ricca memoria visiva funge, si direbbe, da contrappeso all'opzione che emerge con evidenza negli apparati critici. L'introduzione di Mario Richter è centrata su Sainte-Beuve, come si conveniva alla particolare specializzazione dell'autore negli studi sul XIX secolo. È l'impronta propria di questa edizione italiana, rispetto a quella precedente presso Sansoni - la cui introduzione era dovuta alla penna di un secentista, Antoine Adam - e rispetto a quella francese del 2004 presso Laffont, affidata a un grande specialista di Pascal e dell'agostinismo secentesco, Philippe Sellier. Nelle pagine di Mario Richter rivivono il travaglio intellettuale e spirituale del critico romantico, la sua dialettica col proprio tempo, il legame con precedenti e modelli anch'essi ottocenteschi come il Génie du Christianisme di Chateaubriand o NotreDame de Paris di Hugo (dove l'unità dell'intreccio si fa, com'è noto, intorno al centro mitico e simbolico rappresentato dalla cattedrale). La bibliografia stessa è dedicata in prevalenza a Sainte-Beuve, mentre due cronologie ripercorrono, rispettivamente, le date della vita del monastero e quelle dello scrittore. Alla lettura dell'introduzione ben si avverte come la complessa vicenda del libro, del suo contesto, della sua fortuna 
sia in sé un oggetto consistente e avvincente, attraverso il quale guardare, un po' come attraverso il vetro di un acquario, il silenzioso altrove di Port-Royal.

Più che insistere nell'elogio che merita questa splendida edizione, anche sotto il profilo della traduzione cui ha collaborato una squadra ben integrata di cinque traduttrici (Elettra Bordino, Fabiola Baldo, Marina Bernardi, Maria Dario, Alessandra Flores d'Arcais), lasceremo emergere gl'interrogativi che fanno esitare sulla soglia o che affiorano dalla lettura stessa. Come rileggere oggi il Port-Royal? Oggi vuol dire, naturalmente, a un secolo di distanza dal Contre Sainte-Beuve di Proust, tardivamente contraddetto nel 1987 da un Pour Sainte-Beuve di José Cabanis, e quando il genere del portrait littéraire ha addosso un secolo di disaffezione se non di anatema - mentre però ha un così grande successo il genere delle grandi biografie, e narrativa e scrittura della storia celebrano nuove, inedite alleanze, e un bisogno di racconto pervade anche le discipline più severe. Pour et contre Sainte-Beuve era il titolo di un convegno dedicato al Port-Royal quasi vent'anni or sono, proprio a Losanna (mentre l'estremo prolungamento di questa catena di «renversements du pour au contre» sarà un convegno a Parigi nel 2004, presso la Bibliothèque Nationale de France, intitolato fermamente Pour Sainte-Beuve e organizzato da proustiani penitenti).

A Losanna, Jean Molino ha definito felicemente l'opera di Sainte-Beuve, che considerava se stesso un «servitore della scienza», come un tentativo di storia totale ${ }^{1}$. Ebbene, come confrontarsi oggi, in un tempo di crisi della lettura e della totalità, con un libro che ti prende, t'imbarca sul suo lento fiume - si ricorderà che proprio SainteBeuve nelle Pensées de Joseph Delorme esalta l'aspetto fluviale, sinuoso, rispecchiante, osmotico della critica letteraria -, trapassando insensibilmente ogni ragionevole confine; un libro che gioca magistralmente col problema della epoché, ossia della sospensione di un segmento dal tutto, legandola alla storia di un luogo, il monastero, fra il 1204 (anno della sua fondazione) o più autenticamente fra il 1602, anno della elezione a badessa di una bambina chiamata Angélique Arnauld, e il 1710-1713, anni della sua distruzione, ma dilatandola enormemente sul piano orizzontale dei rapporti e delle associazioni, tanto che in questa storia di Port-Royal capitoli digressivi riguardano Montaigne o Molière... Nell'idea di storia totale, sfida all'impossibile, rientra anche l'incrinatura e la faglia: c'è dentro anche la traccia dell'evoluzione vissuta dallo stesso Sainte-Beuve lungo gli oltre vent'anni di gestazione dell'opera, ben manifesta nella diseguaglianza di toni e climi del libro. Così «totale», questo libro, e così incrinato al suo interno, da poter diventare per alcuni lettori - Richter cita Gianfranco Contini (vedi Introduzione, p. LXXIII) - il prediletto, l'unicamente amato, con quella vena di provocazione con cui si predilige ciò che altri non riusciranno ugualmente ad amare.

Il nostro interrogativo ha però una portata più circoscritta. Come rileggere oggi il Port-Royal a fronte di un travolgente sviluppo di studi su giansenismo, agostinismo, logica e retorica gallicana, o su singoli autori tra cui Pascal e Racine? È un controluce accennato nell'apparato critico di questa edizione ed è quello su cui mi pare valga la pena soffermarsi ancora. Richter dà di nuovo la parola a Gianfranco Contini: «E Sainte-Beuve che fa la grandezza di Arnauld, di Nicole, non parliamo di Racine o di Pascal» (p. LXIII). E richiama fuggevolmente l'ostilità votata a Sainte-Beuve da Bremond, forse - segretamente - per la stessa ragione: per aver "fatto" la grandezza di Port-Royal e del suo «gruppo letterario», che Bremond, nel quarto tomo della sua Histoire littéraire du sentiment religieux en France [...], si applica diligentemente a disfare. Non ci sarebbe stato forse lo straordinario affresco della Histoire di Bremond,

(1) J. Molino, 'Port Royal' et la méthode de Sainte-Beuve, in Pour et contre Sainte-Beuve: le 'Port Royal', a cura di J. Mesnard et al., Atti del convegno di Losanna (sett. 1992), Genève, Labor et Fides, 1993, p. 188. 
che ha svelato tanti orizzonti inesplorati della spiritualità francese nel Seicento e, in particolare, ha attribuito il bel nome di «Scuola francese», limpido quanto arbitrario, al filone berulliano e oratoriano, se non si fosse trattato anche di sfatare un mito: il Grand Siècle spirituale come secolo di Port-Royal. Non a caso in quell'affresco la zona più grigia è proprio il tomo quarto, nel quale Bremond fa un ritratto malevolo di Saint-Cyran, dichiara la monotonia del famoso stile di Port-Royal, sottolinea il moralismo e le tendenze anti-mistiche del gruppo giansenista, attribuisce un raffinato panedonismo spirituale, avido di segni, a Pascal nella sua gioia e nelle sue lacrime.

C'è dunque un processo dialettico che ha preso le mosse dal Port-Royal di Sainte-Beuve e in cui in certo senso siamo ancora coinvolti. Molti contemporanei di Sainte-Beuve non capirono la passione da lui dedicata alle ricerche sul monastero. Lamartine se ne meravigliava: avrebbe voluto che Sainte-Beuve si dedicasse a un «grande argomento» (cfr. p. L, nota). Balzac manifestava con virulenza la sua incomprensione; Flaubert, con freddezza, la sua estraneità. Victor Hugo ricevendo l'amico all'Académie gettò sui Solitari di Port-Royal un fascio di luce che parve a Sainte-Beuve meno vero della loro «ombra» (p. LII). Nessuno di quegli scrittori illustri aveva intuito ciò che Sainte-Beuve percepiva così acutamente e che qualcuno in seguito ha chiamato l'enigma di Port-Royal. Lo enuncia efficacemente Philippe Sellier: «Pourquoi énigme? C'est qu'un petit nombre de femmes et d'hommes, presque constamment persécutés et bientôt détruits - au point que de certains de leurs bâtiments ne fut pas laissée pierre sur pierre - ce petit nombre de personnes a exercé une influence inouïe sur la littérature en France, et constitue le groupe intellectuel et spirituel le plus prestigieux, semble-t-il, de toute l'histoire de la culture française» ${ }^{2}$. Un secolo di studi, attraversato anche da intensi dibattiti, e specialmente la massiccia rivisitazione operata negli ultimi decenni del Novecento permettono di prendere le distanze dal giudizio paradossale di Gianfranco Contini: no, non è la penna di Sainte-Beuve ad aver "fatto" la grandezza dei Solitari, così come l'animosità sottile di Henri Bremond non ha avuto l'esito di disfarla. Ė possibile oggi votare al libro di Sainte-Beuve un amore geloso, sceglierlo a livre de chevet, e tuttavia cercare altrove le misure interpretative di quel mondo di cui il grande critico sondava, con trepido sguardo di moralista, insieme la «misère» e la «grandeur».

Enigma. Una rievocazione letteraria come il Port-Royal di Montherlant, una inchiesta sociologica così «parziale, appassionata, politica» come quella del Dieu caché di Goldmann (1955), la sottile e aporetica rilettura del segno nella Critique du discours di Louis Marin (1975), una requisitoria inquietante come quella di Kolakowski in Dieu ne nous doit rien (1997) - per citare solo alcuni notissimi episodi - non hanno contribuito a dissiparlo: piuttosto gli hanno dato spessore. Un romanzo recente, $\mathrm{La}$ blessure et la soif di Laurence Plazenet (Gallimard 2009), ha mostrato ancora come quella densità sia feconda per l'immaginario. Le assemblee delle Società intitolate a Port-Royal nell'austero locale che fu un tempo cappella a Port-Royal de Paris, mirabile nel suo spogliamento, o i convegni presso le antiche Granges hanno insieme il carattere di iniziative scientifiche e di riti ispirati alla pietas. Ma quegli emozionanti convegni si concretizzano poi in volumi che illuminano reti di rapporti, d'influssi indispensabili per una comprensione non solo di Port-Royal ma del cristianesimo e della cultura secenteschi (Port-Royal e l'Oriente; Port-Royal e la filosofia; Port-Royal e le immagini: ecco alcuni degli ultimi titoli). Una intensa attività editoriale continua a svolgersi in più direzioni: lavori vasti e sistematici su aspetti interni alla storia del monastero e del suo gruppo, ad esempio la poesia a Port-Royal (v. Le Chant de la grâce 
di Tony Gheeraert, 2003) o la spiritualità bernardina (Port-Royal et saint Bernard de Clairvaux di Simon Icard, 2010); edizioni di testi nati nell'ambiente di Port-Royal, dalla traduzione delle Confessioni dovuta a Arnauld d'Andilly, alla prima edizione delle Pensées di Pascal confrontata con le Copies che servono di base alle edizioni moderne (recente fatica di Jean-Robert Armogathe), ai capisaldi della memorialistica giansenista come i Mémoires di Louis de Pontis o quelli di Nicolas Fontaine, oggetto, questi ultimi, di una imponente edizione critica ad opera di Pascale Thouvenin (2002). È ridiventato possibile leggere non solo in biblioteca Nicole, grazie a una scelta degli Essais a cura di Laurent Thirouin, e alla ferma analisi critica di Béatrice Guion. Nel solco del magistero di Jean Mesnard e di Philippe Sellier generazioni di studiosi hanno fatto le loro prove, e i più giovani non sono certo i meno attratti dal «faro oscuro» della Solitudine. Leggendo tanti testi editi o riediti recentemente, che Sainte-Beuve conobbe in profondità, si ha come l'impressione di andare a scoprire le carte del suo gioco. Si entra nel suo laboratorio. Si va oltre la sua affascinante mediazione.

Salta agli occhi oggi, rileggendo il capolavoro, come in esso la dimensione più assente sia quella del rayonnement letterario e culturale del gruppo di Port-Royal. Il critico letterario ha fatto come un passo indietro: l'unico vero affondo di natura letteraria è quello che egli compie sulle Provinciales di Pascal. In realtà, una volta ridefinita la categoria di giansenismo, una volta restituito il suo pieno valore a quella di agostinismo che copre territori ben più larghi e investe anche regioni della cultura mondana, l'enigma di Port-Royal cessa di avere i caratteri dell'eccezione: la solitudine si apre da tutte le parti al dialogo. Una mostra a Roma, Il Dio nascosto (2000), con splendidi testi introduttivi di Armogathe e Fumaroli, ricollegando l'«età dell'eloquenza» alla «scuola del silenzio» della pittura ha allargato i contesti e arricchito le coordinate delle grandi famiglie spirituali, tra Italia e Francia, tra gesuiti e gallicani, barocco e classicismo. A Philippe Sellier che ha scritto due volumi su Port-Royal et la littérature (1999-2000), sono stati offerti, quasi in risposta, dei mélanges dal titolo Le rayonnement de Port-Royal (2001); e mi pare che in questo titolo ci sia l'essenza di ciò che la critica e la storiografia del Xx secolo, forti delle loro domande inquiete come della loro pazienza analitica e costruttiva, hanno apportato alla conoscenza del monastero. In pratica oggi gli studi sul gruppo di Port-Royal innervano la conoscenza critica del Seicento francese.

Tutto ciò, mi pare, rende oggi più liberi nel chiedere al Port-Royal di Sainte-Beuve qualcosa che nessun successivo bilancio degli studi, sempre mobile e provvisorio, potrebbe venire a modificare. Un libro come questo chiede di esser letto dal punto di vista dell'universale. Le posizioni datate, le affermazioni criticamente inaccettabili (quanto spazio dedicato, quanta positivistica diligenza impiegata a demitizzare il miracolo della «sainte Épine»!) sono quello che Chateaubriand chiamava, nella Vie de Rancé, il tremito del tempo. Ma è intramontabile il sofferto dibattito intimo con cui Sainte-Beuve accompagna la narrazione, e quel rimettere continuamente in questione persino le proprie motivazioni di storiografo. È coinvolgente lo scrupolo di verità, insieme con una fiducia nell'eroismo umano abbastanza grande da non temere di denunciare senza ritegno le debolezze - «quell'insieme di buono e cattivo che è, precisamente, la vita» (p. LXI). Il monumento elevato qui a Port-Royal è, di fatto, meno trionfalistico di certi accenti apologetici risuonati più tardi. La pensosità dello scrittore nasce dalla posta che è realmente in gioco in questa fatica di una vita: «chi non conosce Port-Royal non conosce l'umanità» sono parole di Royer-Collard', cui

(3) Citate da Sainte-Beuve nel Discorso promunciato all'Accademia di Losanna: cfr. Port-Royal,

p. 18 dell'edizione che presentiamo. 
Richter fa eco in una prospettiva più storicistica: Port-Royal è «un osservatorio privilegiato dell'intera realtà moderna nelle sue origini» (p. LII). La «storia totale» diviene piuttosto, per un intimo classicismo che lo scrittore romantico custodisce in sé, una inchiesta sull'umano. E si comprende allora la tensione del moralista tra il fatto ed il mito, lo scetticismo e la fede, la poetica delle rovine e il canto della permanenza.

Consigliamo, a chi s'immerge nel Port-Royal, una breve lettura preliminare: quella dell'incipit dei Mémoires di Fontaine. Il più umile dei Solitari, che invecchia lontano da Port-Royal, scrive per ricordare. Fa freddo nella stanza della sua memoria, alle cui pareti egli appende i ritratti dei grandi maestri, per intrattenersi con loro e costruire così una diga contro l'erosione del tempo, che è anche un'erosione dell'anima. Scrivendone, gli sembra che i volti cari si animino e vengano a rianimarlo: ricomincia una conversazione, nel clima assorto di una memoria affettiva che ha in realtà una portata morale e tocca, con la mediazione delle immagini, la sfera dell'assoluto. Parliamo di Fontaine e siamo entrati forse, insensibilmente, nel Port-Royal di SainteBeuve. Tant'è vero che la critica, la storiografia sono un lento fiume che avvolge il proprio oggetto, si adatta ad esso e vive nel rispecchiarlo. 